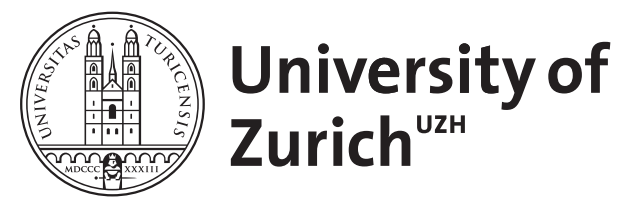

\title{
The fuzziness of giant planets' cores
}

\author{
Helled, Ravit ; Stevenson, David
}

\begin{abstract}
Giant planets are thought to have cores in their deep interiors, and the division into a heavyelement core and hydrogen-helium envelope is applied in both formation and structure models. We show that the primordial internal structure depends on the planetary growth rate, in particular, the ratio of heavy elements accretion to gas accretion. For a wide range of likely conditions, this ratio is in one-to-one correspondence with the resulting post-accretion profile of heavy elements within the planet. This flux ratio depends sensitively on the assumed solid-surface density in the surrounding nebula. We suggest that giant planets' cores might not be distinct from the envelope and includes some hydrogen and helium, and the deep interior can have a gradual heavy-element structure. Accordingly, Jupiter's core may not be well defined. Accurate measurements of Jupiter's gravitational field by Juno could put constraints on Jupiter's core mass. However, as we suggest here, the definition of Jupiter's core is complex, and the core's physical properties (mass, density) depend on the actual definition of the core and on the planet's growth history.
\end{abstract}

DOI: https://doi.org/10.3847/2041-8213/aa6d08

Posted at the Zurich Open Repository and Archive, University of Zurich ZORA URL: https://doi.org/10.5167/uzh-143118

Journal Article

Published Version

Originally published at:

Helled, Ravit; Stevenson, David (2017). The fuzziness of giant planets' cores. Astrophysical Journal Letters, 840(1):4.

DOI: https://doi.org/10.3847/2041-8213/aa6d08 


\title{
The Fuzziness of Giant Planets' Cores
}

\author{
Ravit Helled ${ }^{1,2}$ and David Stevenson ${ }^{3}$ \\ ${ }^{1}$ Institute for Computational Science, University of Zurich, Zurich, Switzerland \\ ${ }^{2}$ School of Geosciences, Tel-Aviv University, Tel Aviv, Israel \\ ${ }^{3}$ Division of Geological and Planetary Sciences, Caltech, Pasadena, CA, USA \\ Received 2017 January 30; revised 2017 March 23; accepted 2017 April 4; published 2017 April 26
}

\begin{abstract}
Giant planets are thought to have cores in their deep interiors, and the division into a heavy-element core and hydrogen-helium envelope is applied in both formation and structure models. We show that the primordial internal structure depends on the planetary growth rate, in particular, the ratio of heavy elements accretion to gas accretion. For a wide range of likely conditions, this ratio is in one-to-one correspondence with the resulting post-accretion profile of heavy elements within the planet. This flux ratio depends sensitively on the assumed solid-surface density in the surrounding nebula. We suggest that giant planets' cores might not be distinct from the envelope and includes some hydrogen and helium, and the deep interior can have a gradual heavy-element structure. Accordingly, Jupiter's core may not be well defined. Accurate measurements of Jupiter's gravitational field by Juno could put constraints on Jupiter's core mass. However, as we suggest here, the definition of Jupiter's core is complex, and the core's physical properties (mass, density) depend on the actual definition of the core and on the planet's growth history.
\end{abstract}

Key words: planets and satellites: composition - planets and satellites: interiors

\section{Introduction}

The formation of a giant planet in the core accretion model can be divided into three main phases (Pollack et al. 1996; Helled et al. 2014):

1. Phase-1. Primary core/heavy-element accretion. During this phase, the core accretes solids within its feeding zone, until it reaches isolation mass. The envelope's mass (hydrogen-helium) is much smaller than the heavyelement mass $M_{Z}$. Solid material could arrive as small bodies (pebbles) or as very large bodies (merging embryos/giant impacts), but here we focus on the intermediate case, which is also the best-studied caseplanetesimal accretion. During this early phase, the protoplanet's atmosphere is close to hydrostatic equilibrium and merges smoothly with the low-density nebula at the Hill Sphere, and most of the accreted planetesimals reach the surface of a well-defined heavy-element core.

2. Phase-2. Slow envelope/gas accretion. During this phase, the solid accretion rate decreases, and the gas (hydrogen and helium) accretion rate increases until the envelope accretion rate exceeds the core (solid) accretion rate. The envelope's growth expands the planet's feeding zone and thus allows more planetesimals to be accreted but at a slow rate.

3. Phase-3. Rapid gas accretion. Once the protoplanet reaches the crossover mass $\left(M_{\mathrm{H}-\mathrm{He}}=M_{\mathrm{Z}}\right)$, gas accretion rate continuously increases and exceeds the solid accretion rate until the disk can no longer supply gas fast enough to maintain equilibrium and keep up with the planetary contraction. After reaching that point, hydrodynamic gas accretion begins.

The disruption of planetesimals breaks them to small particles. Above $\sim 1 M_{\oplus}$, the accretional energy (per unit-mass) exceeds the latent heat of vaporization of rock. The energy required to keep the gaseous atmosphere hot is small by comparison. At the earliest stages ( $\sim$ a few $M_{\oplus}$ or less), the gaseous mass in the atmosphere is small and increases with time, in order to maintain hydrostatic equilibrium. As a result, the heavy-element mass influx greatly exceeds the gas-mass influx and the rock and ice still form a core, albeit very hot, even supercritical (with a small amount of gas mixed in). At a later stage (phase-2), the main focus here, the ratio of gas-mass influx to heavy-element influx rises, eventually reaching and exceeding unity. Most of the mass ( $\sim 90 \%$ of the planet) accumulates during phase-3 (runaway) when the gas influx is fast and thus greatly exceeds the heavy-element influx.

The planetary primordial internal structure depends on various physical processes and model assumptions. While various detailed numerical models of giant planet formation exist, there is an advantage to using simple models in order to get a feeling for the problem. Therefore, our arguments build up on the model of Stevenson (1982) where the surface temperature of a growing core is $\sim 4000 \mathrm{~K}$ at one Earth mass $\left(M_{\oplus}\right)$ and is increasing as $\propto M^{2 / 3}$. The gas's density at that surface depends on the accretion rate and opacity; for an accretion timescale of $10^{6}$ years, it is $\sim 10^{-3} \mathrm{~g} \mathrm{~cm}^{-3}$, suggesting that the atmospheric mass is very small but the ram pressure exerted on incoming planetesimals of velocity $v$ is $\sim 1000$ bar $\left(v / 10 \mathrm{~km} \mathrm{~s}^{-1}\right)^{2}$, sufficient for planetesimal disruption.

We claim that the post-accretion structure of the planetary core accretion directly reflects the history of relative accretion (gas accretion rate to heavy-element accretion rate):

$$
Z(m) \approx\left(d M_{Z} / d t\right) /\left(d M_{\text {tot }} / d t\right) \quad \text { at } \quad M(t)=m,
$$

where $Z(m)$ is the post-accretion ratio of heavy elements to gas (hydrogen and helium) at the radius that contains mass $m . M_{Z}$ is the total mass of heavy elements, and $M_{\text {tot }}$ is the total mass, both evaluated at the time when the total mass is $m \ll$ a Jupiter-mass. This means the heavy-element distribution postaccretion (at an age of several million years) is a direct reflection of how the planet accreted. This is not necessarily the present-day structure because convective mixing can spread the 
heavy elements further (Leconte \& Chabrier 2012; Vazan et al. 2016). Accordingly, the primordial fuzziness of the core of a giant planet is a direct consequence of the gradual change in the accretion ratio of heavy elements and hydrogen and helium over time. This would only go away if one thought (unrealistically) that the planet abruptly switched from pure heavy elements to pure gas accretion at some time. This is impossible since quasi-hydrostatic equilibrium mandates gas accretion when heavy elements are accreted.

Our suggestion is linked to the following criteria:

1. A monotonic decrease of $\left(d M_{Z} / d t\right) /\left(d M_{\text {tot }} / d t\right)$ with increasing $m$.

2. Heating due to the accretion causes a decrease in local gas density that is less than the increase of density arising from the injection of heavy elements into the same mass of gas.

3. Deposition of incoming heavies occurs over a range of radii that corresponds to a small mass fraction of the planet at that time.

4. Deposition occurs at a radius that encloses most of the mass at that time.

The first criterion is required for gravitational stability of the resulting Z-profile. It is satisfied by all standard models of planetary growth. The second depends on the dimensionless parameter $A$ defined by the ratio of density change due to heating to the density change due to increase in the mean molecular weight:

$$
A=v^{2} / 2 C_{p} T
$$

where $v$ is the velocity at the location where planetesimal break-up occurs, $T$ the ambient temperature at that radius, and $C_{p}$ the specific heat. This assumes the heavies have a mean molecular weight far larger than that of a hydrogen-helium mixture. A necessary condition for vigorous mixing is $A$ substantially greater than unity. In that case, the consequence of an incoming planetesimal would be a rising plume of very hot but enriched gas rather than the "local" deposition of new heavy material. Even at $A \sim 1$, mixing is not highly efficient. Actual models predict $A<1$, though not by a large amount. Typically, $v<10 \mathrm{~km} \mathrm{~s}^{-1}$ (break-up occurs at $\sim 10^{10} \mathrm{~cm}$ when the total mass is $\sim 10 M_{\oplus}$ ).

Criteria 3 and 4 are only needed to sharpen the validity of Equation (1). They were typically satisfied, but the essence of our main claim would still apply even if they were not strictly satisfied. However, validity of our suggestion regarding $Z(m)$ does not automatically lead to a prediction for the final $Z$ profile, which depends on the specific conditions under which the giant planet has formed, in particular, the solid-surface density $\sigma$.

\section{Jupiter's Primordial Internal Structure}

In order to demonstrate the sensitivity of the internal structure to the formation history, we use two formation models for Jupiter: Model-1 with $\sigma=10 \mathrm{~g} \mathrm{~cm}^{-2}$ and Model-2 with $\sigma=6 \mathrm{~g} \mathrm{~cm}^{-2}$ at 5.2 au with planetesimal sizes of $100 \mathrm{~km}$ taken from Lozovsky et al. (2017).

The calculation begins with a small core $\left(\sim 0.1 M_{\oplus}\right)$. The heavy-element distribution is determined by following the planetesimals' trajectory as they pass through the envelope

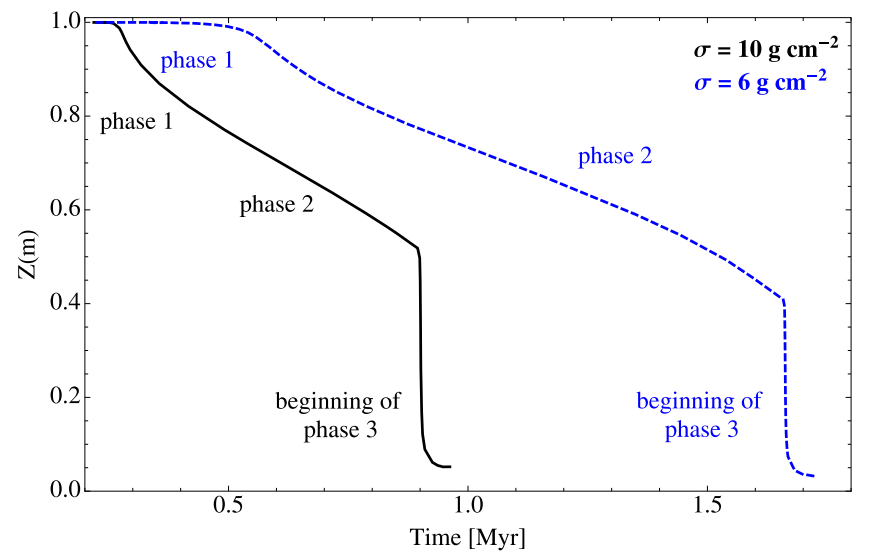

Figure 1. $Z(m)$ vs. time for the two cases $\sigma=10 \mathrm{~g} \mathrm{~cm}^{-2}$ (black) and $\sigma=6 \mathrm{~g} \mathrm{~cm}^{-2}$ (dashed blue).

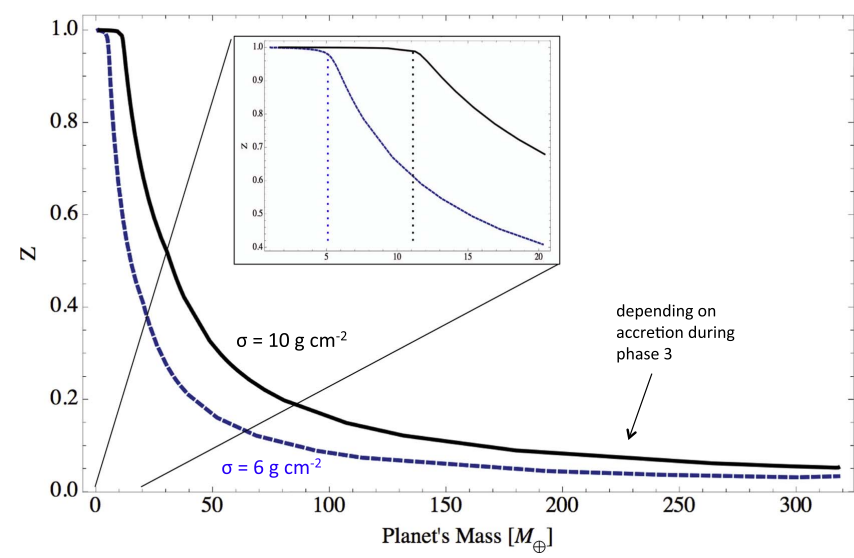

Figure 2. $Z$ vs. planetary mass for $\sigma=6 \mathrm{~g} \mathrm{~cm}^{-2}$ (dotted) and $\sigma=10 \mathrm{~g} \mathrm{~cm}^{-2}$ (solid). This demonstrates the dependence of the planetary composition on the relative accretion rate (see Equation (1)). A zoom-in of $Z$ vs. time up to a mass of $20 M_{\oplus}$ is shown in the small panel.

accounting for gas drag and gravitational forces. The effects of heating, ablation, and planetesimal fragmentation and settling due to saturation are also included. In Model-1, crossover is reached after $0.94 \mathrm{Myr}$, when $M_{Z}=16 M_{\oplus}$, while for Model-2, crossover time is $1.54 \mathrm{Myr}$ and $M_{Z}=7.5 M_{\oplus}$. Crossover time is calculated assuming that all the accreted planetesimals reach the core, while the deposition of heavies into the envelope is expected to decrease the time by a factor of a few (Venturini et al. 2016).

Figure 1 shows $Z(m)$ versus time for the two models up to crossover time. Jupiter's formation in Model-1 is faster due to the higher $\sigma$, and $M_{Z}$ is higher than in the case of $\sigma=6 \mathrm{~g} \mathrm{~cm}^{-2}$ where the primordial structure is more gradual in composition. Once the heavy elements are deposited in the atmosphere, the temperature increases significantly due to the change in opacity, and heavy elements evaporate in the envelope. In Model-2, the innermost region of the planet does not have a core+envelope structure as in Model-1, but the concentration of heavy elements is very high, mimicking a diluted core.

Figure 2 shows $Z$ versus total planetary mass throughout the planetary growth until Jupiter's mass is reached. It is interesting to note the large difference in the predicted composition for planets with $\sim 20 M_{\oplus}$ for the two models-while one is composed of mostly heavy elements, and is more similar to Uranus/Neptune, the low- $\sigma$ case results in a planet with a much lower mean density, as several of the observed exoplanets. In 


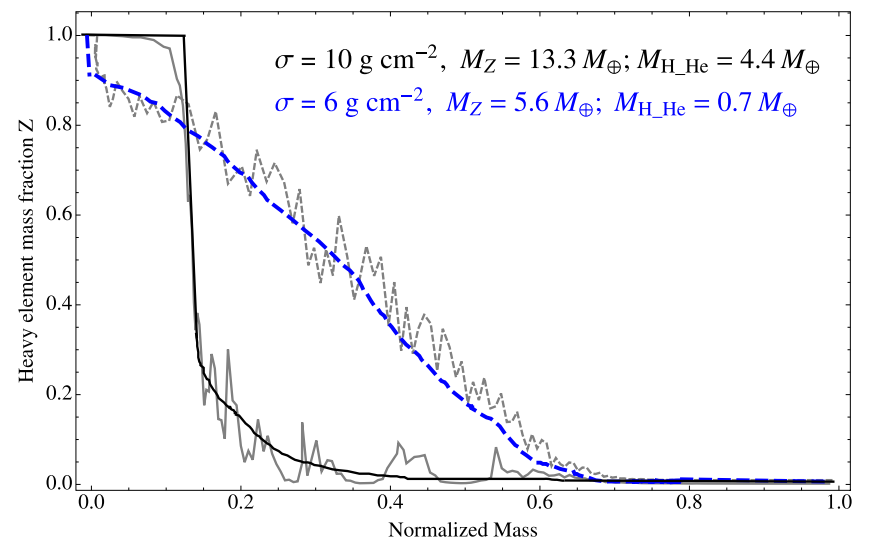

Figure 3. $Z$ vs. normalized mass for $\sigma=10 \mathrm{~g} \mathrm{~cm}^{-2}$ (solid black) and $\sigma=6 \mathrm{~g} \mathrm{~cm}^{-2}$ (dotted blue) at time of $0.66 \mathrm{Myr}$. The two different distributions persist during the planetary formation. Unlike the model of Lozovsky et al. (2017), we do not assume that mixing and settling take place during the formation process. The gray curves show the original distribution before smoothing is applied. The black and blue curves give guidelines to the expected distribution in the two cases.

Model-1, the protoplanet consists of mostly heavy elements up to $M \sim 11 M_{\oplus}$, then, as the planet's mass increases, more $\mathrm{H}-\mathrm{He}$ is accreted and $Z$ drops. Once runway gas accretion begins, $Z$ decreases even further, and the planet's composition is determined by the composition of the accreted gas and the solid accretion rate during phase-3.

In Model-2, $M_{Z}$ is smaller and the growth is slower. Therefore, the protoplanet consists of pure- $Z$ up to a smaller mass of $\sim 5 M_{\oplus}$ and $Z$ drops faster than in Model-1. In both cases, when the planet reaches a Jupiter-mass, $Z$ is nearly solar. Model-1 represents a standard core+envelope configuration, while Model-2 results in a less-conventional picture of Jupiter's interior with a more gradual internal structure, and its innermost regions can be viewed as an extended core. The latter corresponds to an onion-like internal structure as suggested by Stevenson (1982). If Jupiter's interior is found to be more similar to that of Model-2, it would suggest that the local surface density was comparable to the one predicted by the minimum mass solar nebula (MMSN). During phase-2, the accreted material is mostly $\mathrm{H}-\mathrm{He}$ gas, but also has a small portion of heavy elements resulting in a slight increase of $M_{Z}$ when the planet reaches runaway (phase-3).

Figure 3 shows $Z$ versus normalized mass for the two models at the time of $0.66 \mathrm{Myr}$. At this time, in Model-1, the total mass is almost $18 M_{\oplus}$, with $13 M_{\oplus}$ of heavies, while for Model- $2 M_{Z}=5.6 M_{\oplus}$ out of a total mass of $6.3 M_{\oplus}$. Since the growing planet in the two models has a different formation path, there is no point in comparing their $M_{\mathrm{Z}} / M_{\mathrm{H}-\mathrm{He}}$, but instead the actual distribution of the heavy elements. It is clear from the figure that Model-1 has a core+envelope structure, while in Model-2 the heavy elements are distributed gradually and there is no discontinuity in the density profile.

If one defines the core of the planet by region with say, $Z>0.7$, the core is more extended (up to $20 \%$ of the total mass) and also consists of non-negligible amounts of hydrogen and helium. This leads to a lower core density than in the core +envelope configuration. Also, the gradual heavy-element distribution would inhibit large-scale convection. This can affect the heat transport efficiency, and therefore also its longterm evolution and current-state internal structure.
The presented heavy-element distribution assumes that the composition gradients persist during the formation process even in convective regions. Note that this is different from the model of Lozovsky et al. (2017), where the regions with composition gradients that were found to be convective according to the Ledoux convection criteria were assumed to homogenize instantaneously due to mixing. However, homogenizing convective regions with composition gradients requires a fairly long time $\sim 10^{7}-10^{9}$ years $\gg$ formation timescale (see Vazan et al. 2016). The exact timescale depends on the mixing model, particularly, on the mixing length when using mixing length theory. This requires a detailed study on mixing in giant planets, which is beyond the scope of this Letter, and we hope to address this topic in detail in future research.

For the arguments presented here, the core could still be quite well defined as the central concentration of heavy elements, provided it is central enough. For example, suppose an accretion model (perhaps subsequently modified by convection) predicts that $Z(m)=(2 a / \sqrt{\pi})\left(1-Z_{e}\right) \exp \left(-a^{2} m^{2} / m_{c}^{2}\right)+Z_{e}$, where $Z_{e}$ is $Z$ in the envelope value (e.g., $3 \%$, corresponding to $10 M_{\oplus}$ ). The central contribution of heavy elements is then indeed $m_{c}$ (provided the parameter $a$ is not a great deal less than unity; it cannot be more than $\sqrt{\pi} / 2$ and presumably everyone would be satisfied with that as the "core mass." In such a case, there would be no sharply defined core region.

Jupiter's primordial internal structure can differ from its present one. Several processes can affect the internal structure during the long-term evolution. The miscibility of heavy elements in metallic hydrogen allows core erosion (Wilson \& Militzer 2012), the extent of which is determined by the ability of overlying thermal convection to do the gravitational work needed to erode the core and develop the gradual redistribution as the planet cools and contracts. Vigorous mixing is expected to occur mostly in the first $10^{8}$ years because that is when the planet cools efficiently and its interior is less degenerate (Guillot et al. 2004; Vazan et al. 2016). It seems that in the inner regions where the compositional gradient is steep, the mixing may be inefficient. The efficiency of double-diffusive convection is imperfectly understood and needs further investigation (e.g., Leconte \& Chabrier 2012).

\subsection{Heavy-element Accretion during Runaway Gas Accretion}

In both models we present, $M_{Z}$ at crossover is smaller than the standard estimates of $20-40 M_{\oplus}$ of heavy-element mass for Jupiter from interior models (e.g., Guillot 2005). Even for $\sigma=10 \mathrm{~g} \mathrm{~cm}^{-2}$, which is $\sim 3 \times \mathrm{MMSN}, M_{Z} \sim 16 M_{\oplus}$. This is even more prominent for $\sigma=6 \mathrm{~g} \mathrm{~cm}^{-2}$ with $M_{Z}=7.5 M_{\oplus}$. Clearly, in order to further enrich Jupiter with heavy elements, more solids (planetesimals) must be accreted during phase-3. Calculating the solid accretion rate during this phase is not trivial since on one hand the planetary feeding zone increases rapidly, while on the other hand, the growing planet is expected to scatter many of the planetesimals. The exact solid accretion rate during phase-3 is unknown and depends on several physical processes including the accretion morphology, gap formation, planetesimal dynamics, etc.

Alternatively, Jupiter's envelope could be relatively depleted in heavy elements and have nearly solar composition at least with respect to the elements that are in planetesimals. This leaves unanswered the puzzle of why the observed heavy elements in Jupiter's atmosphere exceed solar by a factor of 
$\sim 3$. It is an open issue as to whether these were delivered by planetesimals. A configuration of a core and a proto-solar composition of the envelope has also been suggested by interior modelers (e.g., Hubbard \& Militzer 2016). In such a scenario, the accreted gas during runaway is expected to be depleted in heavy elements (Helled \& Lunine 2014). In any case, it is clear that the solid accretion rate during phase- 3 is crucial for determining the final planetary composition. Once the planet reaches a mass of $\sim 20 M_{\oplus}$ most of the accreted mass is hydrogen and helium gas, and as a result, the composition of the gas and/or the solid accretion rates during that stage are crucial. Therefore, in order to link giant planet bulk composition with origin, we must have a better understanding of the late accretion. This should include the predicted composition gas and the composition (and rate) of accreted solids and their formation location in the solar nebula.

Another way to further enrich the planet with heavies is migration. If Jupiter had formed farther out and then migrated inward, the feeding zone is never depleted and the total heavyelement mass can be $\sim 40 M_{\oplus}$ (Alibert et al. 2005). This is different for in situ formation models of Jupiter in which the planet depletes its feeding zone. Further heavy-element enrichment can also be a result of accretion of gas that is enriched with volatiles. Indeed, Guillot \& Hueso (2006) suggested that Jupiter's envelope enrichment with noble gases could be due to late accretion of nebular gas depleted in $\mathrm{H}-\mathrm{He}$.

\section{Conclusions and Discussion}

Giant planets' interiors can be more complex than a simple division of a core+envelope. We show that the final composition and structure depend on the conditions available for the planet's formation location and growth history. Therefore, Jupiter could consist of a central region in which the heavy-element concentration decreases gradually outward. While the models we present do not necessarily represent Jupiter's origin, they clearly demonstrate the complexity in modeling Jupiter's origin and the challenges in linking giant planet formation and internal structure.

We present the sensitivity of the derived planetary composition and internal structure to the model parameters and emphasize the importance of understanding the late formation stages (phase-3), which determine the final composition of the planetary envelope. However, the primordial distribution of heavy elements could change during the planetary long-term evolution due to core erosion and/or convective mixing (e.g., Guillot et al. 2004; Wilson \& Militzer 2012; Vazan et al. 2016). We suggest that the mass and composition of giant planet cores depend on their exact formation history. We find that the lower $\sigma$ is, the more likely it is to have composition gradients in the deep interior. While the layers in which the gradient is moderate can mix by convection, the inner region, where the gradient is steep and the heavyelement mass fraction is high, is expected to remain stable. This configuration could provide the starting point for developing layered convection (Leconte \& Chabrier 2012). Giant planet formation models should be developed further and be combined with the evolution models to investigate whether the material is mixed during their long-term evolution. It is also desirable to further investigate accretion of both gas and solids and their mixing during phase-3.

There is no unique definition for giant planets' cores. How should the core be defined? Is it by the physical state of the material? Its density? Is it the requirement for a density discontinuity? Is it the stability against convection, or is it the fraction of high- $Z$ material that has to be sufficiently large? Defining it by physical state makes little sense-we do not know whether a heavy-element-enriched central region would be solid or liquid (it depends on the temperature, which is unknown, and possibly much higher than the adiabat that is typically assumed), and in any event, such a definition would de-emphasize the thing we most want to know: the extent to which there is an excess of heavy elements near the planet's center. For the same reason, emphasizing the existence or absence of a density discontinuity makes no sense and is not physically meaningful given the quantum mechanical calculations that predict miscibility. This is the essential difference between giant and terrestrial planets, where the sharpness of the core-mantle boundary is a direct consequence of the immiscibility of the core and mantle regions.

We thank Peter Bodenheimer, Michael Lozovsky, Allona Vazan, Tristan Guillot and an anonymous referee for valuable comments. R.H. acknowledges support from SNSF grant 200021_169054.

\section{References}

Alibert, Y., Mordasini, C., Benz, W., \& Winisdoerffer, C. 2005, A\&A, 434,343

Guillot, T. 2005, AREPS, 33, 493

Guillot, T., \& Hueso, R. 2006, MNRAS, 367, L47

Guillot, T., Stevenson, D. J., Hubbard, W. B., \& Saumon, D. 2004, in Jupiter The Planet, Satellites and Magnetosphere, ed. F. Bagenal, T. E. Dowling, \& W. B. McKinnon (Cambridge: Cambridge Univ. Press), 35

Helled, R., Bodenheimer, P., Podolak, M., et al. 2014, in Protostars and Planets VI, ed. H. Beuther et al. (Tucson, AZ: Univ. Arizona Press), 643

Helled, R., \& Lunine, J. 2014, MNRAS, 441, 2273

Hubbard, W. B., \& Militzer, B. 2016, ApJ, 820, 80

Leconte, J., \& Chabrier, G. 2012, A\&A, 540, A20

Lozovsky, M., Helled, R., Rosenberg, E. D., \& Bodenheimer, P. 2017, ApJ, 836, 227

Pollack, J. B., Hubickyj, O., Bodenheimer, P., et al. 1996, Icar, 124, 62 Stevenson, D. 1982, P\&SS, 30, 755

Vazan, A., Helled, R., Podolak, M., \& Kovetz, A. 2016, ApJ, 829, 118

Venturini, J., Alibert, Y., \& Benz, W. 2016, A\&A, 596, A90

Wilson, H., \& Militzer, B. 2012, PhRvL, 108, 111101 